University of Nebraska - Lincoln

DigitalCommons@University of Nebraska - Lincoln

8-1997

\title{
Spurious Electrons in Electron Spectrometers and their Effect on Differential Electron Impact Ionization Cross-Section \\ Measurements
}

\author{
M. Eugene Rudd \\ University of Nebraska - Lincoln, erudd@unl.edu
}

Follow this and additional works at: https://digitalcommons.unl.edu/physicsrudd

Part of the Physics Commons

Rudd, M. Eugene, "Spurious Electrons in Electron Spectrometers and their Effect on Differential Electron Impact Ionization Cross-Section Measurements" (1997). M. Eugene Rudd Publications. 40.

https://digitalcommons.unl.edu/physicsrudd/40

This Article is brought to you for free and open access by the Research Papers in Physics and Astronomy at DigitalCommons@University of Nebraska - Lincoln. It has been accepted for inclusion in M. Eugene Rudd Publications by an authorized administrator of DigitalCommons@University of Nebraska - Lincoln. 


\title{
Spurious electrons in electron spectrometers and their effect on differential electron impact ionization cross-section measurements
}

\author{
M. E. Rudd \\ Department of Physics and Astronomy, University of Nebraska, Lincoln, Nebraska 68588-0111
}

(Received 20 March 1997; accepted for publication 19 May 1997)

\begin{abstract}
The effect of the nonideal response characteristics of electrostatic analyzers is described and equations are derived, which allow a calculation of the distortion that this causes in measurements of angular and energy distributions of electrons from atomic collisions, especially in electron impact. Examples are given that show how a contamination as small as $10^{-5}$ from spurious electrons generated inside an analyzer can explain the peak seen at zero angle in some angular distribution measurements and the filling in of the minimum in the energy distributions in others.

(C) 1997 American Institute of Physics. [S0034-6748(97)04908-3]
\end{abstract}

\section{INTRODUCTION}

Electrostatic analyzers have been widely used as electron energy spectrometers in various applications for several decades. Ideally, they pass no electrons except those in a narrow range of energies, the position of which can be swept through the spectrum. But, in fact, they depart from this ideal and allow the detection of a small fraction of electrons having energies higher or lower than the nominal pass energy. Froitzheim, Ibach, and Lehwald ${ }^{1}$ described the effect of reflection of low-energy electrons from the outer electrode of a $127^{\circ}$ analyzer and showed the effect of machining a fine sawtooth surface on the inside of the outer plate to reduce the effective reflection coefficient. Irby $\mathrm{et} \mathrm{al} .^{2}$ and Bernardi and Meckbach ${ }^{3}$ have described the effect that fast electrons striking the back plate of a parallel-plate analyzer could have on the detected electron spectra from ion-atom collisions.

Measurements of the spectra of electrons emitted during electron-impact ionization are especially susceptible to these effects because of the presence of elastically scattered electrons and incident electrons that have lost energy due to excitation or ionization of the target. The purpose of this paper is to draw attention to this problem and to analyze its effects. The treatment here is general and applies to any type of deflection analyzer. Differences among analyzers or the presence of a focusing system at the entrance to the analyzer will affect the response function but not the equations to be derived in Sec. III. Although the emphasis here will be on electron impact ionization, the results can easily be modified to treat other cases such as ionization by ion impact.

\section{SPECTROMETER RESPONSE FUNCTION}

In order to assess the effect of spurious electrons on the spectrum, one must measure the response of the spectrometer to monoenergetic electrons over a wide range of analyzer settings both above and below the energy of the main peak. Froitzheim et al. ${ }^{1}$ presented such a graph for $7 \mathrm{eV}$ incident electrons and found spurious peaks of $2-4 \times 10^{-3}$ times the height of the main peak. A response curve given by Bernardi and Meckbach $^{3}$ has a spurious peak with a height of $1-8$ $\times 10^{-4}$ relative to the main peak. Two additional examples of such response curves, shown in Figs. 1(a) and 1(b), have contamination fractions in the range $10^{-4}-10^{-2}$. These plots, normalized to unity at the main peaks, are the response functions $f\left(W / W^{\prime}\right)$, where $W$ is the analyzer pass energy and $W^{\prime}$ is the energy of the entering electrons. These examples show that while the details of the response curves depend on the type of analyzer used, the basic features, namely a large, narrow main passband with a smaller but nonzero response on either side, are similar. If the electron beam used to record the response function has an appreciable energy width, the sharper features of the curve will be broadened but the basic shape is not affected. Bernardi and Meckbach $^{3}$ have found that while the positions of the features of the response curve depend only on $W / W^{\prime}$, the relative amount of the contamination increases with $W^{\prime}$. For simplicity, and due to the lack of systematic data, we will ignore this dependence in the analysis.

By calculating electron trajectories inside the analyzer, one can often identify the origin of various features of the response curve. For example, for the parallel-plate analyzer of Fig. 1(b), the region from 0 to 0.5 results from electrons with energies greater than twice the pass energy $W$, which strike the back plate producing slow secondary electrons and reflected electrons, some fraction of which finds its way to the detector. From 0.5 to 0.8 , there is a succession of peaks resulting from the beam striking the four field-straightener frames. The peaks immediately below and above the main peak are from electrons striking the edges of the small depression made for inserting the disk containing the exit slit. The higher end of the response curve results primarily from electrons with energies less than $W$ striking the inside of the front plate between the entrance and exit slits. The geometry of the particular analyzer, of course, will affect the exact form of the response function.

Methods have been employed to reduce the number of spurious electrons, such as (1) coating the inside surfaces of the analyzer with a material such as soot to absorb incident electrons more effectively; (2) milling a sawtooth surface on the electrodes, as mentioned above; (3) cutting a slot in the back plate to let higher energy electrons pass on through the analyzer; (4) replacing the back plate with a hightransparency grid; (5) providing a second-stage analyzer to better isolate the main peak; and (6) providing a potential hill after the exit slit of the analyzer to suppress spurious lowenergy electrons. Although these and other measures may 

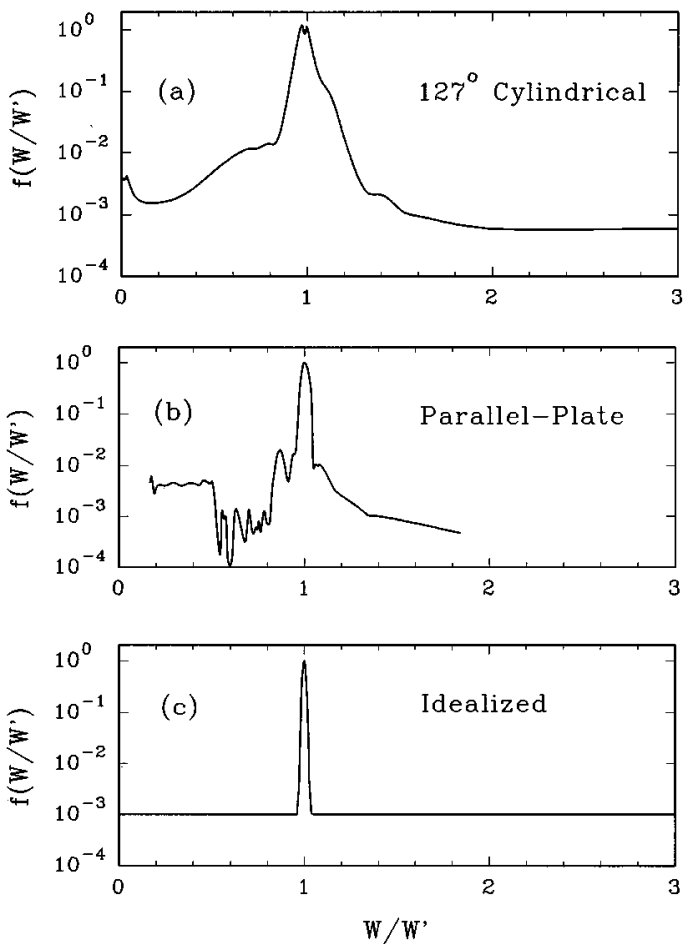

FIG. 1. Response functions of electrostatic analyzers to monoenergetic electrons of energy $W^{\prime}$. W is the pass energy of the analyzer. (a) Measurements on a $127^{\circ}$ cylindrical analyzer with $5.7 \%$ full width at half-maximum resolution. (b) Measurements on a parallel-plate analyzer with $4.6 \%$ resolution. (c) An idealized response curve with $2 \%$ resolution.

reduce the problem, they do not eliminate it. As will be shown, even contamination fractions as low as $10^{-5}$ can have a serious effect on the measured spectra and angular distributions. Unfortunately, none of the published measurements of electron ionization spectra contain analyzer response functions and other data from which one could estimate the needed corrections.

\section{EFFECT OF SPURIOUS ELECTRONS ON THE SECONDARY ELECTRON DISTRIBUTION}

The electron energy spectrum from electron collisions varies with the emission angle $\theta$, the incident energy $T$, and the target gas. In general, there is a continuum with a zeroenergy peak, a binary encounter peak if $\theta<90^{\circ}$, and a minimum followed by a rise to a peak at $T-I$, where $I$ is the first ionization potential of the target gas. Above the ionization limit, there is a series of sharp lines from scattered primary particles, which have lost energy to excitation of various states of the target. Above $T-E$, where $E$ is the first excitation potential, the spectrum drops to zero, but at $W=T$ there is a large peak due to the elastic scattering of electrons from the incident beam. Figure 2 shows a schematic diagram of the spectrum in which the elastic peak is represented by a delta function, and the infinite number of excitation lines have been replaced by a single delta function for simplicity. Because of the nonideal characteristics of analyzers, electrons from any part of the spectrum can contaminate the measurement of the cross section at any other part of the spectrum.

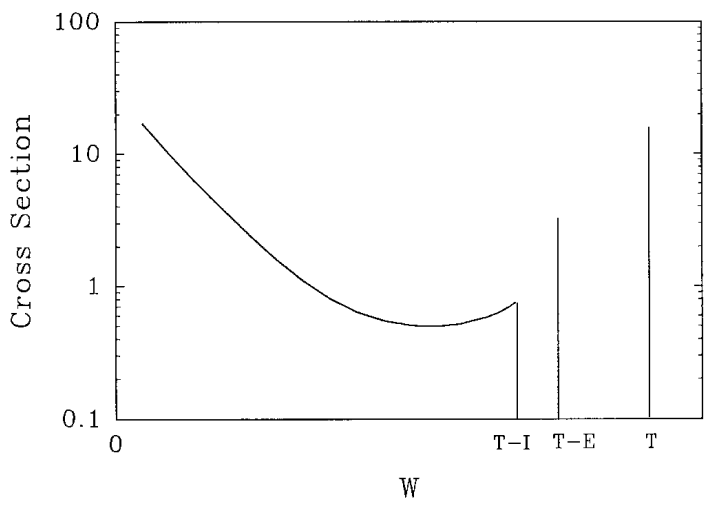

FIG. 2. Schematic diagram of the energy spectrum of electrons at a fixed angle resulting from electron impact on a target gas. The continuum consists of scattered incident electrons, which have lost energy to ionization in addition to secondary electrons ejected from the target. The single line at $T-E$ represents the infinite number of lines resulting from electrons that have lost energy by excitation to various states of the target. The line at $T$ is the elastic peak.

In a measurement of the angular and energy distribution of electrons, the measured doubly differential cross section (DDCS) due to ionization, $\sigma_{i}^{\text {meas }}(W, \theta)$, is given by

$$
N=n(l \Delta \Omega)_{\mathrm{eff}} N_{0} \Delta W \sigma_{i}^{\text {meas }}(W, \theta),
$$

where $N$ is the number of ejected and scattered electrons of energy $W$ at the angle $\theta$ when $N_{0}$ incident electrons of energy $T$ pass through the target gas of number density $n$, $(l \Delta \Omega)_{\text {eff }}$ is the effective value of the product of the beam length viewed and the solid angle accepted by the analyzer, ${ }^{4}$ and $\Delta W$ is the width of the energy window of the analyzer when set for a pass energy $W$.

Taking into account the contamination by spurious electrons, the equation may be rewritten

$$
\begin{aligned}
N= & n(l \Delta \Omega)_{\mathrm{eff}} N_{0} \int_{0}^{\infty} f\left(W / W^{\prime}\right)\left[\sigma_{i}\left(W^{\prime}, \theta\right)+\sigma_{\mathrm{ex}}(\theta)\right. \\
& \left.\times \delta\left(T-E-W^{\prime}\right)+\sigma_{\mathrm{el}}(\theta) \delta\left(T-W^{\prime}\right)\right] d W^{\prime} .
\end{aligned}
$$

Here, $\sigma_{\mathrm{el}}(\theta)$ is the differential elastic scattering cross section, $\sigma_{\text {ex }}(\theta)$ is the differential excitation cross section, and $\sigma_{i}\left(W^{\prime}, \theta\right)$ is the DDCS for the production of electrons of energy $W^{\prime}$, including scattered primary electrons as well as secondary electrons ejected from the target. The quantity $f\left(W / W^{\prime}\right)$ is the fraction of electrons of energy $W^{\prime}$ that register counts when the spectrometer is set to pass electrons of energy $W$. This function is the response function of the spectrometer described in the previous section. Delta functions are used to represent the elastic and excitation peaks, as in Fig. 2.

To proceed further, we write the analyzer response function as the sum of two parts,

$$
f\left(W / W^{\prime}\right)=f_{\text {peak }}\left(W / W^{\prime}\right)+f^{\prime}\left(W / W^{\prime}\right),
$$

where $f_{\text {peak }}$ is the value of the function within the narrow transmission window and $f^{\prime}$ is the function over the rest of the spectrum. Using Eq. (3) and equating the right-hand sides of Eqs. (1) and (2), 


$$
\begin{aligned}
\sigma_{i}^{\text {meas }}(W, \theta)= & \sigma_{i}(W, \theta) \\
& +\frac{1}{\Delta W}\left[\int_{0}^{T-I} f^{\prime}\left(\frac{W}{W^{\prime}}\right) \sigma_{i}\left(W^{\prime}, \theta\right) d W^{\prime}\right. \\
& \left.+f^{\prime}\left(\frac{W}{T-E}\right) \sigma_{\mathrm{ex}}(\theta)+f^{\prime}\left(\frac{W}{T}\right) \sigma_{\mathrm{el}}(\theta)\right] .
\end{aligned}
$$

The integral of the term with $f_{\text {peak }}$ yields the true DDCS $\sigma_{i}(W, \theta)$, while the integral with $f^{\prime}\left(W / W^{\prime}\right)$ is the first of the three terms inside the brackets. These terms represent the contributions to the measured cross section from the contamination by ionization, excitation, and elastic scattering, respectively.

This analysis differs from that of Irby et al. ${ }^{2}$ in that we treat the contamination as an addition to the main signal rather than as a multiplicative factor. This seems to be preferable since the main signal comes from one narrow part of the spectrum while the contamination results from all of the rest of the spectrum and the two are not necessarily proportional.

The width of the energy window is $\Delta W=R_{A}$ (W $\left.+E_{\text {acc }}\right)$, where $R_{A}$ is the fractional analyzer resolution and $E_{\text {acc }}$ is the energy by which the electrons have been accelerated before entering the analyzer. In most applications, $E_{\text {acc }}$ is either zero or is held constant at a few electron volts. Even without knowing the form of $f\left(W / W^{\prime}\right)$, it is clear from Eq. (4) that the narrower the energy window, the greater is the contribution of the contamination to the measured cross section. Thus, if $E_{\text {acc }}$ is held constant, the contamination increases as $W$ decreases, a result first pointed out by Irby. ${ }^{2}$ In spite of this, however, the effect of contamination is not usually apparent at low energies since as $W \rightarrow 0$, the ionization cross section increases about as fast as the contamination.

If we multiply both sides of Eq. (4) by $d \Omega=2 \pi \sin \theta$ $d \theta$ and integrate from 0 to $\pi$, we get

$$
\begin{aligned}
\sigma_{i}^{\text {meas }}(W)= & \sigma_{i}(W)+\frac{1}{\Delta W}\left[\int_{0}^{T-I} f^{\prime}\left(\frac{W}{W^{\prime}}\right) \sigma_{i}\left(W^{\prime}\right) d W^{\prime}\right. \\
& \left.+f^{\prime}\left(\frac{W}{T-E}\right) \sigma_{\mathrm{ex}}+f^{\prime}\left(\frac{W}{T}\right) \sigma_{\mathrm{el}}\right]
\end{aligned}
$$

where $\sigma_{i}(W)$ is the singly differential cross section (SDCS) for secondary electron production, and $\sigma_{\mathrm{ex}}$ and $\sigma_{\mathrm{el}}$ are the total excitation and elastic scattering cross sections.

To do the convolution integrals in Eqs. (4) and (5), it is necessary to know the analyzer response function. Since none of the published cross-section measurements is accompanied by such information, we will make the simplifying approximation that $f^{\prime}\left(W / W^{\prime}\right)=f_{c}$, where $f_{c}$ is a constant. This idealized function is shown in Fig. 1(c) for the case $R_{A}=0.02$ and $f_{c}=10^{-3}$. Then, the DDCS in Eq. (4) becomes

$\sigma_{i}^{\text {meas }}(W, \theta)=\sigma_{i}(W, \theta)+\frac{f_{c}}{\Delta W}\left[\sigma_{i}(\theta)+\sigma_{\mathrm{ex}}(\theta)+\sigma_{\mathrm{el}}(\theta)\right]$,

and Eq. (5) for the SDCS reduces to

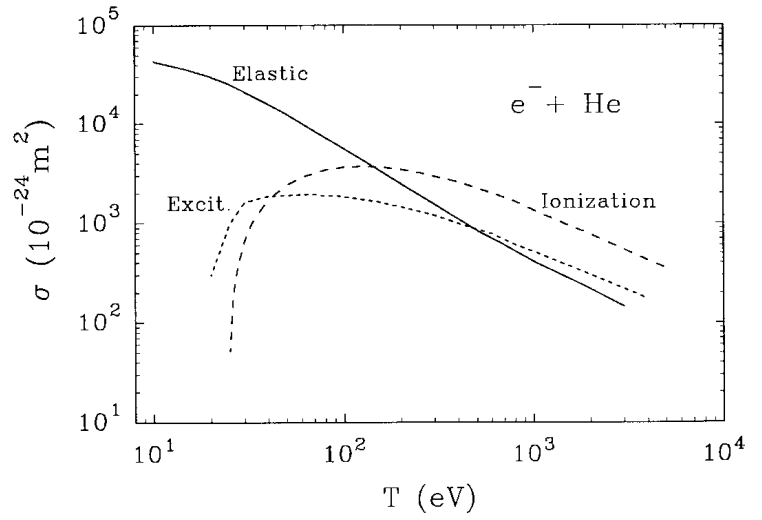

FIG. 3. Total cross sections for ionization, elastic scattering, and excitation in $e^{-}+$He collisions used in the calculation of the effects of analyzer contamination.

$$
\sigma_{i}^{\text {meas }}(W)=\sigma_{i}(W)+\frac{f_{c}}{\Delta W}\left[2 \sigma_{i}+\sigma_{\mathrm{ex}}+\sigma_{\mathrm{el}}\right] .
$$

The factor 2 comes in because we count both the ejected secondary electrons and the scattered primary electrons, leading to double counting of the ionizing collisions.

The equations derived here for electron impact may also be used for the electron spectra from ion or neutral impact if the excitation and elastic terms are omitted, as well as the factor 2 with the total ionization cross sections. The contamination problem for ion impact is, therefore, less important than for electron impact.

\section{EXAMPLES OF THE EFFECT OF CONTAMINATION}

We can use Eq. (6) to calculate the effect of the contamination on the DDCS and Eq. (7) for the SDCS. While only the total cross sections (TCSs) for ionization, excitation, and elastic scattering are needed for the latter, the former requires differential cross sections (i.e., angular distributions). Figure 3 shows the three TCSs for the case of a helium target. Ionization data were taken from Shah et al., ${ }^{5}$ excitation data from Register, Trajmar, and Srivastava ${ }^{6}$ and from de Heer and Jansen, ${ }^{7}$ and elastic cross sections also from Register et $a{ }^{6}{ }^{6}$ Ionization is the most important process above about $150 \mathrm{eV}$, while the elastic cross section dominates at lower energies.

Differential data are more difficult to obtain. Figure 4 shows some data for $500 \mathrm{eV} e^{-}+\mathrm{He}$. The ionization data were obtained by integrating the doubly differential binary encouter-Bethe (BEB) model of Rudd ${ }^{8}$ and Kim and Rudd ${ }^{9}$ over $W$ for many angles. Differential elastic cross sections have been measured for some targets over limited angular or incident energy ranges. The curve in Fig. 4 is a smoothed average of data from Bromberg, ${ }^{10}$ and from Sethuraman, Rees, and Gibson. ${ }^{11}$ Since differential excitation cross sections are not available for the targets and energies used for illustration here, the calculations were made by assuming that the angular distribution of the excitation cross section is the same as that for elastically scattered electrons and normalized to the known total cross section. As justification for this choice, note in Fig. 4 that the $80 \mathrm{eV}$ data of Chutjian and 


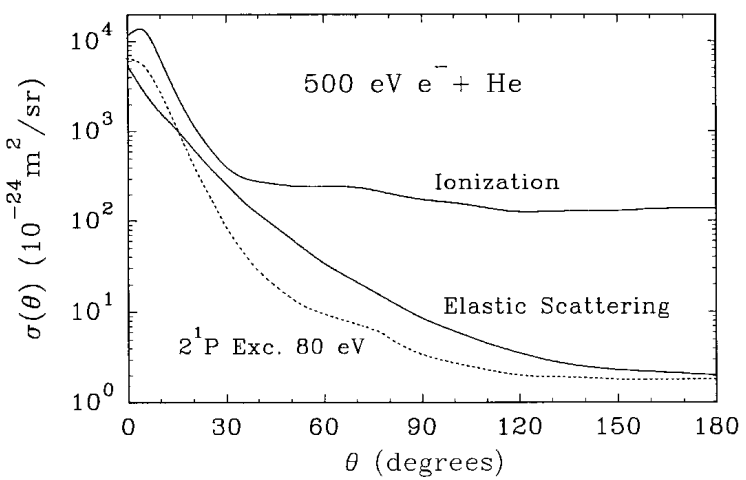

FIG. 4. Angular distributions of electrons from ionization and elastic scattering in $500 \mathrm{eV} e^{-}+\mathrm{He}$ collisions. The dashed line indicates the angular distribution of the cross sections for $2^{1} P$ excitation of helium by $80 \mathrm{eV}$ electrons.

Srivastava ${ }^{12}$ on excitation to the $2^{1} P$ level of helium has an angular distribution not greatly different from the elastic curve.

The assumptions and approximations made in deriving the equations and the lack of data on $R_{A}, f_{c}$, and $\sigma_{\text {ex }}(\theta)$ make it impossible to calculate the exact effect of the contamination on any particular published data. The following examples, therefore, are only representative of the effects of contamination using reasonable values of the parameters. Nevertheless, they indicate quite clearly that contamination of this type can explain some puzzling aspects of the data and some of the discrepancies among the measurements.

\section{A. Spurious peak in the forward direction}

Measurements of electron impact ionization by several experimenters ${ }^{13}$ have exhibited a strong peak in the forward direction, which is not expected according to theoretical treatments. Oda and Nishimura ${ }^{14}$ found that the peak could be reduced or eliminated by biasing at the analyzer exit, but the cause was never determined. Using Eq. (6) and assuming $R_{A}=0.02, E_{\mathrm{acc}}=0$, and $f_{c}=10^{-5}$, we get the results shown

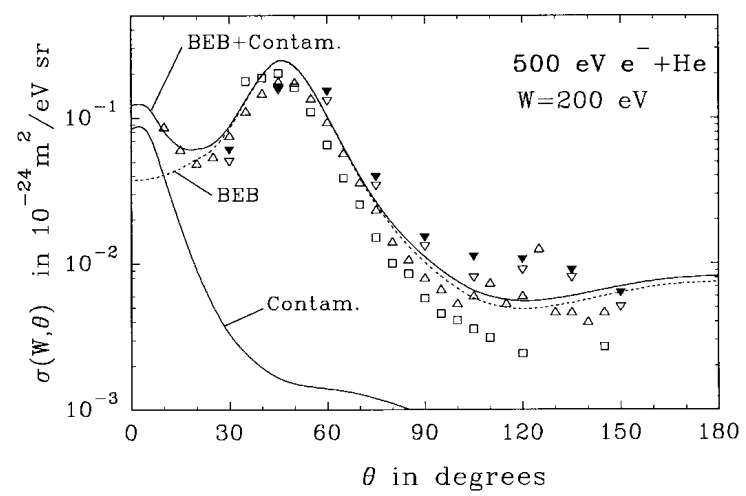

FIG. 5. The effect of analyzer contamination on the angular distribution of $200 \mathrm{eV}$ electrons from $500 \mathrm{eV} e^{-}+\mathrm{He}$ collisions. $\square$, Oda and Nishimura (Ref. 17); $\triangle$, Avaldi et al. (Ref. 16); $\nabla$, Peterson, Beaty, and Opal (Ref. 18 ); $\boldsymbol{\nabla}$, Sethuraman, Rees, and Gibson (Ref. 11); dotted line, calculations using the BEB model of Rudd (Ref. 8) and Kim and Rudd (Ref. 9); solid lines, contamination and the sum of the contamination and the BEB with $R_{A}=0.02, E_{\mathrm{acc}}=0$, and $f_{c}=10^{-5}$.

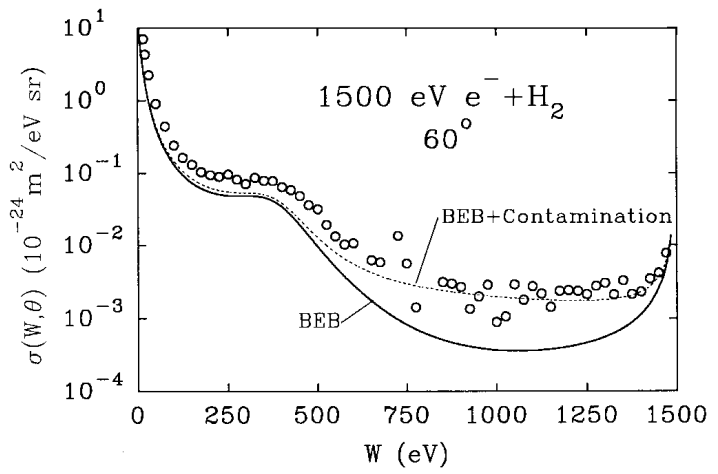

FIG. 6. The effect of analyzer contamination on the energy distribution of electrons detected at $60^{\circ}$ from $1500 \mathrm{eV} e^{-}+\mathrm{H}_{2}$ collisions. $\mathrm{O}$, Rudd et al. (Ref. 15); solid line, BEB; dotted line, BEB plus contamination calculated with $R_{A}=0.01, E_{\mathrm{acc}}=0$, and $f_{c}=5 \times 10^{-5}$.

in Fig. 5. A slightly modified version of the BEB model ${ }^{8,9}$ was used to compare to the data. This shows that a contamination as small as $10^{-5}$ can account for this spurious forward peak.

\section{B. Filling in of the minimum}

In reporting experimental data ${ }^{15}$ on $\mathrm{H}_{2}$, we pointed out that the level region where a minimum is expected in the spectra at backward angles was likely due to a background of spurious electrons, even though a subtraction of backgrounds had been made. Figures 6 and 7 show that this discrepancy between the model and the data in the region of the minimum of the spectrum can be explained by an analyzer contamination with $f_{c}=1.5 \times 10^{-5}$ in one case and $5 \times 10^{-5}$ in the other. Since differential elastic scattering and excitation data were not available for $\mathrm{H}_{2}$ at $1500 \mathrm{eV}$, we made the approximation that the total of the cross sections for ionization, elastic scattering, and excitation was twice the ionization cross section. Since the contamination is due to electrons coming from the target gas itself, it is now clear that subtracting backgrounds taken without target gas cannot correct for this kind of spurious signal.

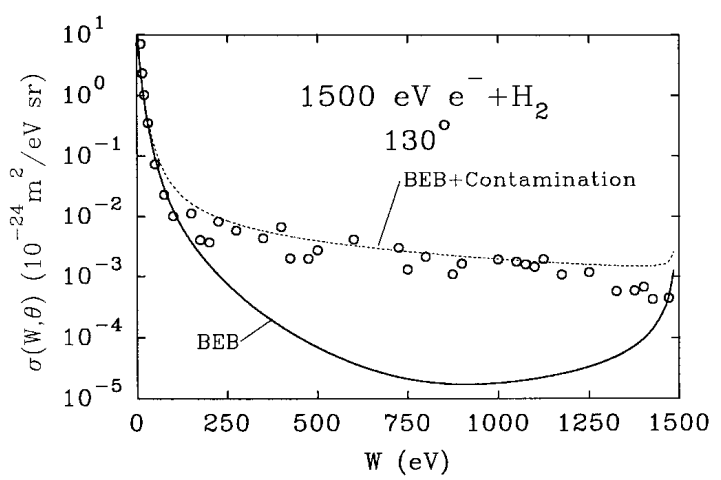

FIG. 7. Same as Fig. 6 for electrons ejected at $130^{\circ}$. 


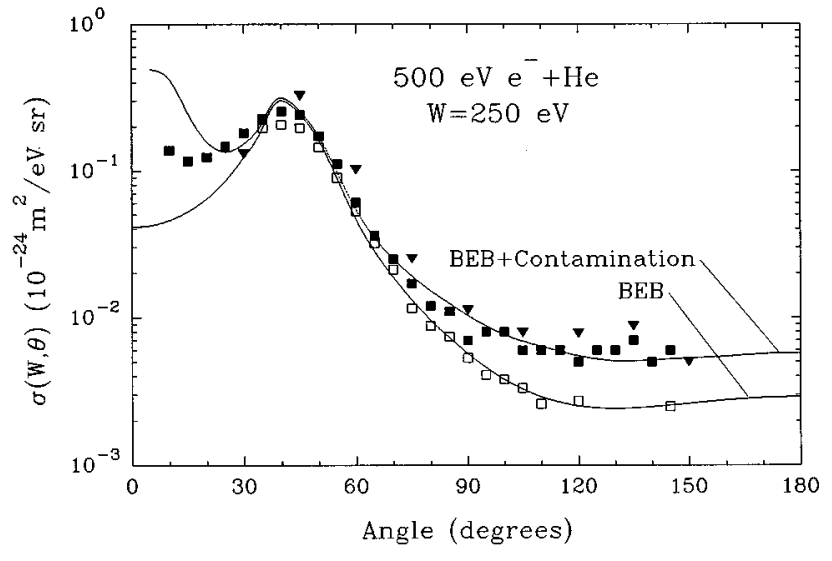

FIG. 8. Graph showing how analyzer contamination could cause the discrepancy in the angular distribution of electrons measured by different investigators. $\square$, Oda and Nishimura (Ref. 17); $\mathbf{\square}$, Avaldi et al. (Ref. 16) and $\boldsymbol{\nabla}$, Sethuraman, Rees, and Gibson (Ref. 19). $R_{A}=0.02, E_{\mathrm{acc}}=0$, and $f_{c}$ $=10^{-4}$.

\section{Discrepancies among data measured by different investigators}

In some cases, discrepancies among measurements made by different investigators appear to be due to analyzer contamination. In Fig. 8, the cross section measurements of Sethuraman, Rees, and Gibson ${ }^{11}$ and Avaldi et al. ${ }^{16}$ are larger than those of Oda and Nishimura ${ }^{17}$ by about a factor of 2 at large angles. Calculations using the BEB model and the contamination equations with $R_{A}=0.02$ and $f_{c}=10^{-4}$ show how this discrepancy can arise. Contamination can also explain the forward peak in the data of Avaldi et al. ${ }^{16}$ although with these parameters the calculated curve overestimates the effect.

Investigators should not only take steps to minimize the contamination produced in their spectrometers but should also make measurements of the analyzer response functions to demonstrate that this type of contamination does not have an appreciable effect on their measurement. If it is not feasible to reduce the contamination to a negligible level, then the equations developed here should be helpful in showing where distortions might appear and how to make suitable corrections.

\section{ACKNOWLEDGMENTS}

The author wishes to thank Timothy Gay for his careful reading of the manuscript and Victor Irby for helpful comments.

${ }^{1}$ H. Froitzheim, H. Ibach, and S. Lehwald, Rev. Sci. Instrum. 46, 1325 (1975).

${ }^{2}$ V. D. Irby, S. Datz, P. F. Dittner, N. L. Jones, H. F. Krause, and C. R. Vane, Phys. Rev. A 47, 2957 (1993); Victor D. Irby, Phys. Rev. A 51, 1713 (1995); V. D. Irby, in Two-Center Effects in Ion-Atom Collisions, edited by Timothy J. Gay and Anthony F. Starace (American Institute of Physics, New York, 1996), p. 237.

${ }^{3}$ G. Bernardi and W. Meckbach, Phys. Rev. A 51, 1709 (1995).

${ }^{4}$ C. E. Kuyatt, in Methods of Experimental Physics, edited by Benjamin Bederson, Wade L. Fite, and L. Marton (Academic, New York, 1968), Vol. 7A.

${ }^{5}$ M. B. Shah, D. S. Elliott, P. McCallion, and H. B. Gilbody, J. Phys. B 21, 2751 (1988).

${ }^{6}$ D. F. Register, S. Trajmar, and S. K. Srivastava, Phys. Rev. A 21, 1134 (1980).

${ }^{7}$ F. J. de Heer and R. H. J. Jansen, J. Phys. B 10, 3741 (1977).

${ }^{8}$ M. E. Rudd, Phys. Rev. A 44, 1644 (1991).

${ }^{9}$ Yong-Ki Kim and M. Eugene Rudd, Phys. Rev. A 50, 3954 (1994).

${ }^{10}$ J. Philip Bromberg, J. Chem. Phys. 61, 963 (1974).

${ }^{11}$ S. K. Sethuraman, J. A. Rees, and J. R. Gibson, J. Phys. B 7, 1741 (1974).

${ }^{12}$ Ara Chutjian and Santosh K. Srivastava, J. Phys. B 8, 2360 (1975).

${ }^{13}$ See, e.g., H. Ehrhardt, K. H. Hesselbacher, K. Jung, M. Schulz, T. Takaat, and K. Willmann, Z. Phys. 244, 254 (1971); Nobuo Oda, Radiat. Res. 64, 80 (1975); T. W. Shyn and W. E. Sharp, Phys. Rev. A 19, 557 (1979); M. A. Bolorizadeh and M. E. Rudd, Phys. Rev. A 33, 882 (1986); L. Avaldi, R. Camilloni, E. Fainelli, and G. Stefani, Nuovo Cimento D 9, 97 (1987).

${ }^{14} \mathrm{~N}$. Oda and F. Nishimura, in Abstracts of the Tenth International Conference on the Physics of Electronic and Atomic Collisions, edited by M. Barat and J. Reinhardt (Commissariat à L'Energie Atomique, Paris, 1977), p. 362; See also M. A. Bolorizadeh and M. E. Rudd (Ref. 13).

${ }^{15}$ M. E. Rudd, K. W. Hollman, J. K. Lewis, D. L. Johnson, R. R. Porter, and E. L. Fagerquist, Phys. Rev. A 47, 1866 (1993).

${ }^{16}$ L. Avaldi, R. Camilloni, E. Fainelli, and G. Stefani (Ref. 13).

${ }^{17}$ Nobuo Oda (Refs. 13 and 14); and (private communication).

${ }^{18}$ W. K. Peterson, E. C. Beaty, and C. B. Opal, Phys. Rev. A 5, 712 (1977).

${ }^{19}$ S. K. Sethuraman, J. A. Rees, and J. R. Gibson, unpublished report, Department of Electrical Engineering and Electronics, University of Liverpool (1972). 